'Servicio de Reumatología e Inmunología Hospital Militar Central, Bogotá, Colombia. ${ }^{2}$ Instituto de Referencia Andino, Bogotá, Colombia. ${ }^{3}$ Instituto de Genética Humana, Pontificia Universidad Javeriana, Bogotá Colombia.

${ }^{4}$ Servicio de Hematología Hospital Militar Central, Bogotá, Colombia.

${ }^{5}$ Unidad de Investigación OralUIBO, Universidad El Bosque, Bogotá, Colombia.

aBacterióloga, Msc, PhD.

'Biólogo, Microbiólogo, PhD. 'Bacterióloga.

${ }^{\mathrm{d}}$ Médico genetista, PhD.

Conflicto de intereses: El proyecto contó con la aprobación de Comité de Investigaciones y Ética del Hospital Militar Central (Código N 2012-085).

Informamos que durante el desarrollo de la presente investigación, ni los autores ni las instituciones que incorporamos incurrimos en ningún tipo de conflicto de intereses.

Financiación: Esta investigación recibió financiación de las instituciones participantes para su realización.

Recibido el 5 de noviembre de 2014, aceptado el 13 de julio de 2015.

Correspondencia a: María Consuelo Romero-Sánchez Transversal 3a № 49-00 Hospital Militar Central. Bogotá, Colombia.

Teléfono: 3486868 spacolombia@gmail.com

\section{Variantes de hemoglobina en una población con impresión diagnóstica positiva para hemoglobinopatías en Colombia}

\author{
CONSUELO ROMERO-SÁNCHEZ ${ }^{1,2,4,5, a}$, \\ ALBERTO GÓMEZ GUTIÉRREZ ${ }^{2,3, \mathrm{~b}}$, YURANI DUARTE ${ }^{1, \mathrm{c}}$, \\ CONSTANZA AMAZO ${ }^{2, c}$, CLARA MANOSALVA ${ }^{2, c}$, LORENA CHILA $\mathrm{M}^{5, \mathrm{c}}$, \\ MARÍA CONSUELO CASAS-GÓMEZ ${ }^{3, c}$, IGNACIO BRICEÑO BALCÁZAR ${ }^{2,3, d}$

\section{Hemoglobin variants in Colombian patients referred to discard hemoglobinopathies}

Background: Oxygen transport is altered in hemoglobinopathies. Aim: To study the distribution of hemoglobinopathies in Andean subjects without African ancestry. Material and Methods: We analyzed blood samples of 1,407 subjects aged 18 to 59 years (58\% females), living in the central Andean region of Colombia, referred to discard hemoglobinopathies. The frequency and type of hemoglobinopathy was established by capillary and agarose gel electrophoresis. Results: The frequency of hemoglobinopathies was $34.5 \%$ and higher among females. The structural variants found were: AS-heterozygous hemoglobin (8.1\%), homozygous SS (3.7\%), heterozygous SC (2.2\%), AC heterozygotes (0.5\%) and heterozygous $A E(0.3 \%)$. Quantitative variants found were $\mathrm{Hb} A$-Beta thalassemia (13.91\%) and $\mathrm{Hb} \mathrm{H}(0.06 \%)$, Beta-thalassemia heterozygotes C (0.88\%), S-Beta thalassemia heterozygotes (6.07\%) and compound heterozygous SC/ Beta thalassemia (0.25\%), with a persistence of fetal hemoglobin 0. Composite thalassemia was also found in $31 \%$. All techniques showed good correlation and capillary electrophoresis demonstrated a greater detection of hemoglobin variants. Conclusions: The frequency of hemoglobin variants in the analyzed population was high, which is an important public health indicator. The most common hemoglobin variant was $\mathrm{HbA} /$ Increased structural $\mathrm{Hb} \mathrm{A} 2$ and the mos frequent structural hemoglobinopathy was sickle cell trait. Capillary electrophoresis can discern any $\mathrm{Hb}$ variants present in the population.

(Rev Med Chile 2015; 143: 1260-1268)

Key words: Hematologic diseases; Hemoglobinopathies; Electrophoresis, agarose gel; Sickle cell trait. 
anemia de células falciformes. Los cromosomas analizados revelaban haplotipos típicos africanos, $55,5 \%$ Bantú, 34,8\% Benin, 4,3\% Senegal y 5,4\% Camerún. Estos datos coinciden con reportes que indican que la mayoría de los esclavos africanos traídos a Colombia proceden de Angola (población Bantú) y la isla de Sao Tomé en el golfo de Benín (África Central Occidental)2.

En el año 2010, el estudio "Determination of the prevalence of hemoglobin $S, C, D$, and $G$ in neonates from Buenaventura, Colombia", determinó el número de individuos para hemoglobina $\mathrm{C}$ con $5,8 \%$, $4,8 \%$ para $S, 0,5 \%$ para $G, 1 \%$ para $D$ y $1 \% S_{-}-C^{3}$.

Alvear y cols. ${ }^{4}$ estimaron la incidencia de las hemoglobinopatías en Cartagena. El diseño metodológico planteó un estudio descriptivo sobre 1.729 muestras de cordón procesadas por isoelectroenfoque. El 4,5\% fueron heterocigotos para la hemoglobinopatía S, $1 \%$ fue heterocigoto para hemoglobina $\mathrm{C}$ y $0,1 \%$ fue doble heterocigoto para S-C.

En varios estudios provenientes de afro-descendientes se encontró alguna variante de hemoglobina, F-C (1,88\%), F-S (4,65\%), S-C (0,06\%), S-S (0,12\%), Bart (0,18\%), F-C/otro (0,12\%). Las muestras de sangre seca de cordón umbilical permiten realizar el estudio por EC y por isoelectroenfoque de las diferentes variantes de hemoglobinas, aplicable al diagnóstico temprano ${ }^{5-8}$.

En el Valle de Aburrá, zona geográfica de Antioquia, Colombia, se realizó por HPLC (high performance liquid chromatography) un estudio de 2.637 muestras de recién nacidos. Se detectaron 93 muestras positivas para hemoglobinopatías, con una prevalencia de $3,7 \%$, siendo la hemoglobina $S$ la más frecuente seguida de $C, D^{9}$.

Las técnicas disponibles para caracterizar cualitativa y cuantitativamente las variantes de hemoglobina son: la electroforesis en gel de agarosa, el isoelectroenfoque, la electroforesis capilar (EC) y la cromatografía líquida. Son técnicas de separación de moléculas a partir de su movilidad según el tamaño o la carga ${ }^{10}$. Una de las variantes es la EC, que se fundamenta en la separación en un tubo de sílice cargado negativamente, mientras que el buffer tiene la carga contraria, lo cual ofrece significativas ventajas como la rápida separación, el volumen de muestra que utiliza y su automatización $^{11}$.

Se han documentado pocos estudios comparativos entre EC y electroforesis en acetato de celulosa a nivel mundial. En Corea se incluyeron pacientes con anemia microcítica hipocrómica con el objetivo de detectar variantes de hemoglobina y talasemias. Ambas técnicas mostraron niveles similares de hemoglobinas y no se observaron diferencias estadísticas significativas. Sin embargo, el artículo recomienda la EC como método de detección rápida, sencilla y fiable para la evaluación de hemoglobinas, aplicable a la población de estudio ${ }^{12}$.

En cuanto a alteraciones cuantitativas de la hemoglobina, el panorama de la documentación no es diferente al de alteraciones cualitativas. En 2009, un grupo de oncohematólogos pediatras y bioquímicos de la Universidad Nacional, sede Bogotá, propuso un método de diagnóstico precoz denominado "balance de cadenas de globina", el cual consiste en el empleo de la PCR (reacción en cadena de la polimerasa) con el objetivo de determinar la presencia de talasemia alfa, la más difícil de diagnosticar precozmente por técnicas convencionales. Esta técnica abre el panorama a un oportuno pronóstico de la enfermedad, mejorando notablemente la calidad de vida del paciente ${ }^{13}$.

Actualmente, las técnicas más utilizadas en nuestro país para la determinación de hemoglobinas son la electroforesis en gel de agarosa y la electroforesis capilar. Sin embargo, a pesar de la pertinencia de estas técnicas en Colombia, aún no se documentan resultados de la situación actual de las hemoglobinopatías en nuestra población general, y de allí surge el interés de analizar a la población andina sin la premisa de la afro-descendencia reciente, así como de describir la experiencia de nuestros laboratorios en el manejo de las diferentes técnicas aplicadas en pacientes con sospecha de hemoglobinopatías.

\section{Materiales y Métodos}

Se realizó un estudio retrospectivo descriptivo no experimental en pacientes con sospecha de hemoglobinopatías que consultaron al Hospital Militar Central (HMC) o referidos al Instituto de Referencia Andino (IRA) provenientes, principalmente, de la región montañosa central de Colombia, entre julio de 2009 y diciembre de 2012. Las muestras cumplían con las siguientes condiciones técnicas específicas: muestras frescas 
anti-coaguladas con EDTA, no hemolizadas y, en caso de estar refrigeradas, debían haber estado en el rango de temperatura de $2-8^{\circ} \mathrm{C}$ hasta un máximo de 5 días. Se excluyeron pacientes con datos clínicos demográficos incompletos.

Para la estratificación de signos, síntomas y diagnósticos de pacientes del Hospital Militar Central se contó con la asesoría de dos hematólogos y un médico internista de la institución (basados en el Nacional Heart, Lung and Blood Institute and The Mayo Clinic Concise Review for Clinicians $)^{14}$, quienes los clasificaron con base en la frecuencia de diagnósticos con los que fueron derivados a su servicio (desde la consulta de medicina general o medicina interna), teniendo en cuenta el abordaje primario con el cual llegaron a consultar en el área de hematología (Tabla 1).

Para la división de la población por regiones se implementó la clasificación de acuerdo a la de investigación de la Unidad de Genética del Instituto Nacional de Medicina Legal y Ciencias Forenses de Colombia ${ }^{15}$. La división territorial adoptada fue la siguiente:
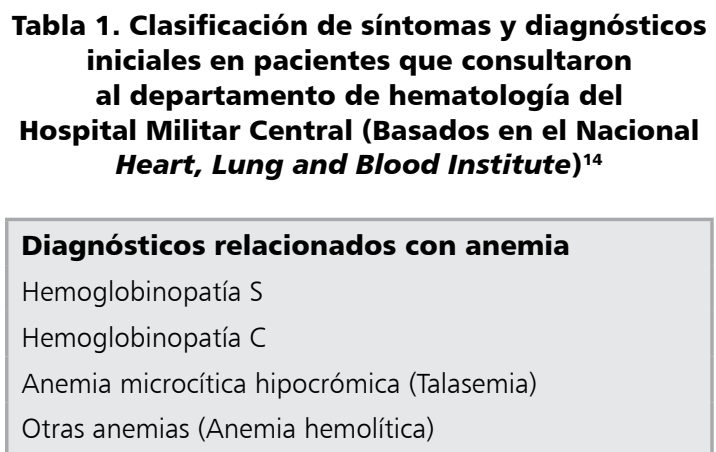

- Región Norte de la costa Pacífica y región marítima de la isla de San Andrés.

- Regiones Andina, Amazónica y Orinoquia.

- Región Suroeste de la región Andina.

- Región de la costa Caribe.

\section{Metodologías utilizadas para la evaluación electroforética}

\section{Electroforesis en gel de agarosa}

La determinación de hemoglobinas se evaluó mediante electroforesis en gel de agarosa en medio alcalino SEBIA ${ }^{\oplus}$ (referencia 4106, París, Francia) y para su confirmación se realizó el corrido en medio ácido SEBIA ${ }^{\oplus}$ (referencia 4108, París, Francia); la separación de moléculas a partir de su movilidad según el tamaño o la carga se realizó en el equipo HYDRASYS 2 Scan de SEBIA ${ }^{\circledast}$ París, Francia, según metodología referida por fabricante; los datos se obtuvieron mediante el software Phoresis $^{\oplus}$ París, Francia.

\section{Electroforesis capilar}

Es una nueva técnica de separación electroforética en medio acuoso, contenido en un pequeño capilar de sílice en presencia de corriente eléctrica en donde la separación electroforética es mucho más rápida y emplea pequeños volúmenes de muestra. Se empleó EC (Minicap) (SEBIA ${ }^{\oplus}$ París, Francia).

\section{Metodología estadística}

No existió técnica limitante de muestreo, ya que se incluyeron, por conveniencia, todas las solicitudes remitidas para la determinación de la hemoglobina y sus variantes durante el período de tiempo definido de julio de 2009 a diciembre de 2012.

Mediante el programa STATA $11^{\circledR}$, se determinó la concordancia entre la electroforesis en gel de agarosa y la electroforesis capilar mediante el coeficiente de correlación de Pearson y el índice Kappa. Adicionalmente, se determinó la frecuencia de las variantes de hemoglobina en las poblaciones estudiadas.

Este estudio se llevó a cabo de acuerdo con los principios éticos comprendidos por la Resolución $\mathrm{N}^{\circ} 008430$ de 1993 del Ministerio de Salud y la Ley 84 de 1989, en la categoría "sin riesgo humano". El proyecto contó con la aprobación de Comité de Investigaciones y Ética del Hospital Militar Central (Código N² 2012-085). 


\section{Resultados}

\section{Análisis de frecuencias}

Para el análisis de frecuencias se tomaron datos de 1.407 individuos, en los cuales el 41,68\% (659) correspondía a hombres; el rango de edad más frecuente fue de 18 a 59 años $(91,97 \%)$ (Figuras 1 y 2) (Tabla 2).

La región 2 representó el mayor número de muestras $(91,90 \%)$ estuvo conformada por Amazonas, Antioquia, Arauca, Boyacá, Caldas, Caquetá, Casanare, Cundinamarca, Huila, Meta, Norte de Santander, Putumayo, Quindío, Risaralda, Santander, Tolima, Valle del Cauca y Vichada. La presencia de variantes de hemoglobina en la población fue de $34,53 \%$ (543 muestras positivas. Los fenotipos de hemoglobinopatías estructurales encontrados fueron: $\mathrm{Hb}$ A-S con $8,09 \%$, S-S con
$3,66 \%, \mathrm{~S}-\mathrm{C}$ con $2,15 \%, \mathrm{~A}-\mathrm{C}$ con $0,50 \%$, A-E con $0,25 \%, A-D$ con $0,12 \%$, S-D con $0,12 \%$ (Tabla 3 ).

Entre las variantes cuantitativas, o síndromes talasémicos, se destacaron: heterocigoto para beta talasemia con presencia del $13,91 \%$, Alfa talasemia con $0,06 \%$. Dentro de este grupo se incluyeron talasemias compuestas: heterocigotos C-beta talasemia con $0,88 \%$, heterocigotos S-Beta talasemia con $6,07 \%$ y heterocigotos compuestos S-C/beta talasemia con $0,25 \%$ (Tabla 4 ). En otras hemoglobinopatías se evidenció persistencia hereditaria de hemoglobina $\mathrm{F}$ en $0,31 \%$.

De acuerdo con los resultados obtenidos, la presencia de hemoglobinopatías se distribuyó: $\mathrm{Hb}$ A-beta talasemia 14,93\%, rasgo falciforme 8,74\%, Hb S-beta talasemia 6,40\%, Hb S-S 3,62\%, Hb S-C $2,35 \%, \mathrm{Hb}$ C-B talasemia con $0,92 \%, \mathrm{Hb} \mathrm{A}-\mathrm{C}$ con $0,57 \%, \mathrm{Hb}$ A-E $0,28 \%$, persistencia hereditaria de

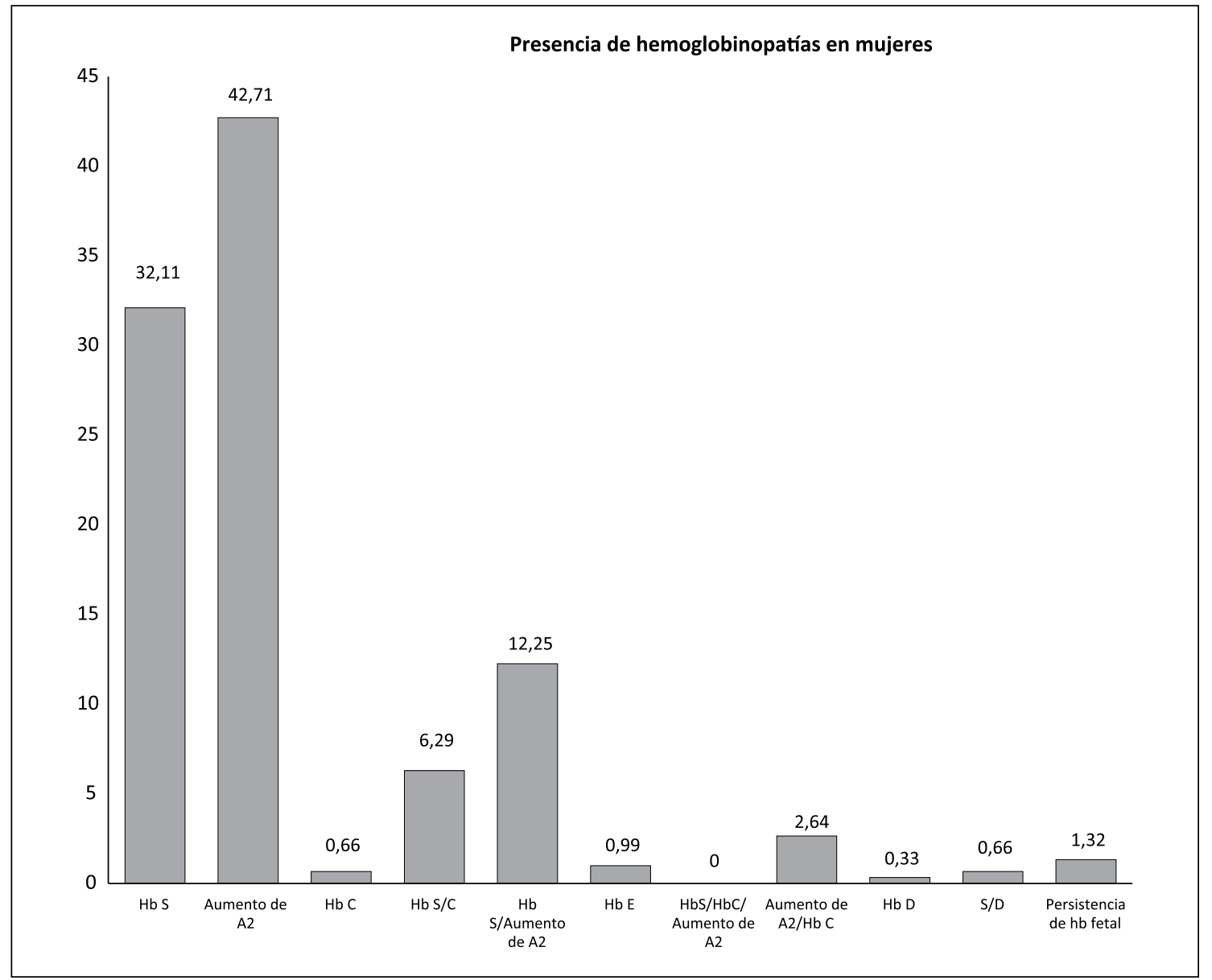

Figura 1. Frecuencia de hemoglobinopatías en mujeres. 


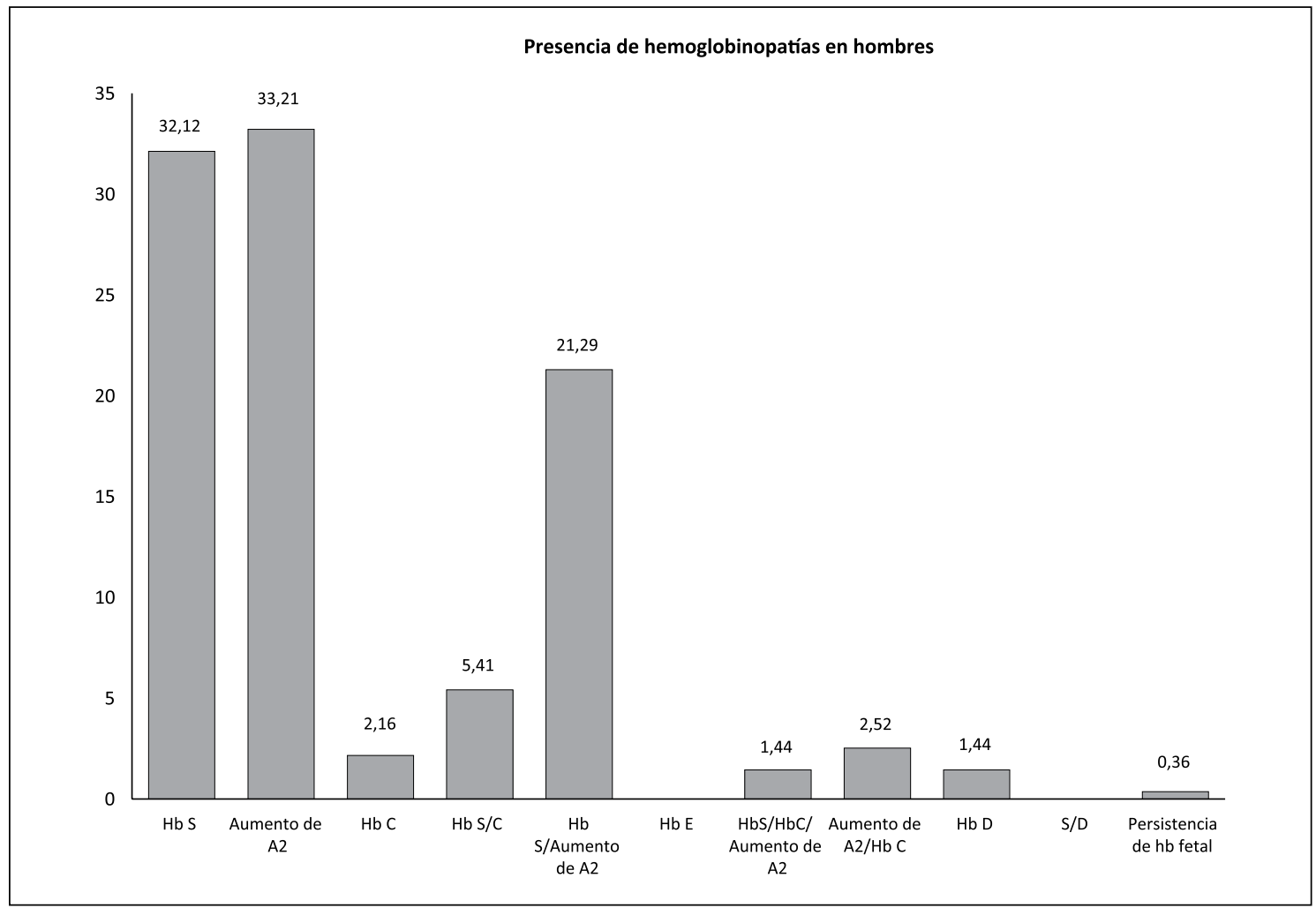

Figura 2. Frecuencia de hemoglobinopatías en hombres.

Tabla 2. Frecuencia de hemoglobinopatías por edad

\begin{tabular}{|lc|}
\hline Rango de edad afectado & Frecuencia \\
\hline Entre 18 y 59 años & $46,93 \%$ \\
\hline Menores de 6 años & $28,91 \%$ \\
\hline Entre 7 y 17 años & $16,13 \%$ \\
\hline Mayores a 60 años & $8,03 \%$ \\
\hline
\end{tabular}

Tabla 3. Frecuencia de hemoglobinopatías estructurales

\begin{tabular}{|ll|}
\hline Rasgo falciforme Hb A-S & $8,09 \%$ \\
\hline Homocigotos S-S & $3,66 \%$ \\
\hline Heterocigotos S-D & $0,12 \%$ \\
\hline Heterocigotos S-C & $2,15 \%$ \\
\hline Heterocigotos A-E & $0,25 \%$ \\
\hline Heterocigotos A-D & $0,12 \%$ \\
\hline Heterocigotos A-C & $0,50 \%$ \\
\hline
\end{tabular}

hemoglobina fetal (PHHF) 0,36\%, Hb S-C/Beta talasemia $0,28 \%$, Hb A-D 0,14\%, Hb S-D 0,14\% y finalmente $\mathrm{Hb} \mathrm{H} \mathrm{0,07 \%} \mathrm{(Tabla} \mathrm{5).}$

\section{Comparación de técnicas para la determinación de hemoglobina}

Para la comparación metodológica se analizaron los resultados de 39 pacientes obtenidos por electroforesis capilar y electroforesis en gel

\section{Tabla 4. Frecuencia de hemoglobinas cuantitativas}

\begin{tabular}{|lr}
\hline Síndromes talasémicos & \\
Heterocigotos A- Beta talasemia & $13,91 \%$ \\
$\mathrm{Hb} \mathrm{H}$ & $0,06 \%$ \\
\hline Talasemias compuestas & \\
Heterocigotos S-Beta talasemia & $6,07 \%$ \\
Heterocigotos compuestos S-C/Beta talasemia & $0,25 \%$ \\
Heterocigotos C-Beta talasemia & $0,88 \%$ \\
\hline
\end{tabular}


de agarosa. El índice Kappa fue de 0,82 con una concordancia esperada de 0,90.

La concordancia para la hemoglobina $\mathrm{F}$ fue de $\mathrm{R}^{2}$ de 0,867 , estadístico $\mathrm{T}=-1,64$ y $\mathrm{p}=0,107$ y para la HB S de $\mathrm{R}^{2}$ de 0,999 , estadístico $\mathrm{T}=-0,257$ y $p=0,797$. En las Figuras 3 y 4 se observa los resultados para la hemoglobina A y A2.

La concordancia encontrada es considerada buena $(0,793)$ de acuerdo con los puntajes establecidos por Landis y Koch. La sensibilidad de las técnicas empleadas fue de $100 \%$ y su especificidad de $91,7 \%$.

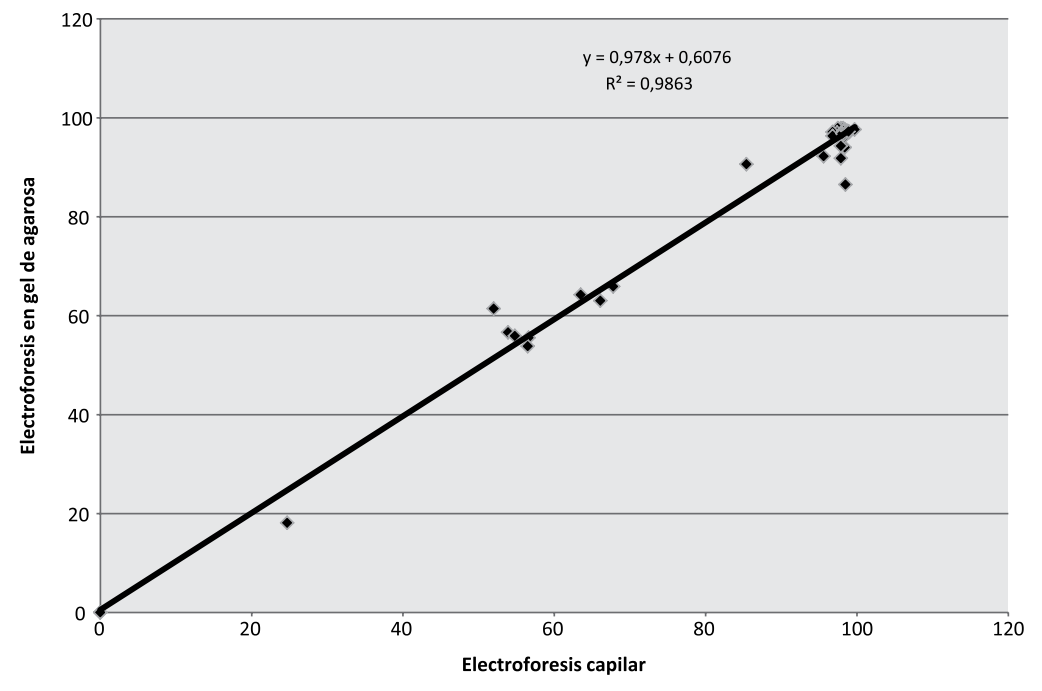

Figura 3. Concordancia $\mathrm{Hb} A \mathrm{R}^{2}$ de 0,986 , estadístico $T=0,36$ y $p=0,71$.

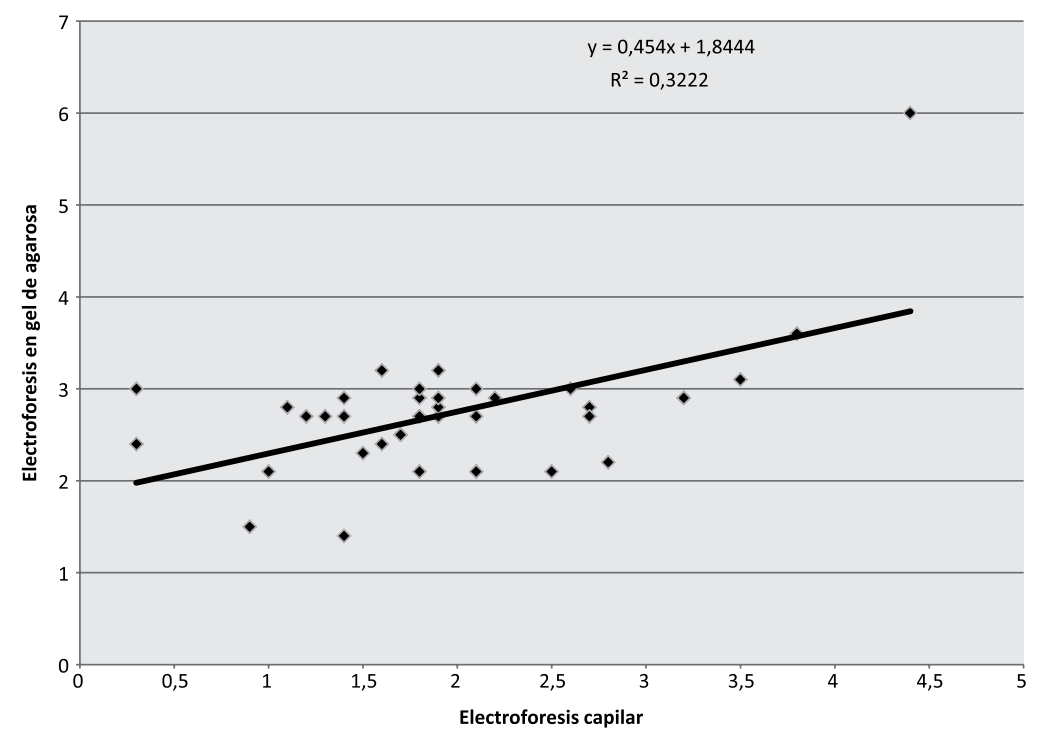

Figura 4. Concordancia hemoglobina $A 2 R^{2}$ de 0,322 , estadístico $T=0,05$ y $p=0,95$. 
Tabla 5. Curso clínico de pacientes con hemoglobinopatías en el Hospital Militar Central

\begin{tabular}{|c|c|c|c|c|c|c|c|c|c|}
\hline \multirow{2}{*}{$\begin{array}{l}\mathbf{n} \\
2\end{array}$} & \multirow{2}{*}{$\begin{array}{c}\text { Hemoglo- } \\
\text { binopatía } \\
\text { Homocigoto } \\
\text { S-S }\end{array}$} & \multicolumn{2}{|c|}{$\begin{array}{l}\text { Diagnóstico con } \\
\text { cuadro clínico }\end{array}$} & \multicolumn{2}{|c|}{$\begin{array}{l}\text { Anemia según } \\
\text { cuadro hemático }\end{array}$} & \multicolumn{2}{|c|}{$\begin{array}{l}\text { Aumento de } \\
\text { reticulocitos }\end{array}$} & \multicolumn{2}{|c|}{$\begin{array}{l}\text { Síntomas de } \\
\text { anemia }\end{array}$} \\
\hline & & $100 \%$ & $\begin{array}{l}\text { Hemoglobi- } \\
\text { nopatía S }\end{array}$ & $100 \%$ & $\begin{array}{l}\text { Normocítica } \\
\text { normocrómica }\end{array}$ & $\begin{array}{l}50 \% \\
50 \%\end{array}$ & $\begin{array}{l}\text { Con aumento } \\
\text { Normal }\end{array}$ & $100 \%$ & Relacionados \\
\hline 50 & $\begin{array}{c}\text { Beta } \\
\text { Talasemia }\end{array}$ & $\begin{array}{l}38 \% \\
36 \% \\
26 \%\end{array}$ & $\begin{array}{l}\text { Talasemia } \\
\text { Diagnóstico } \\
\text { no relacionado } \\
\text { Otras anemias }\end{array}$ & $\begin{array}{r}4 \% \\
10 \% \\
4 \% \\
82 \%\end{array}$ & $\begin{array}{l}\text { Normocítica } \\
\text { normocrómica } \\
\text { Microcítica } \\
\text { hipocrómica } \\
\text { Normocítica } \\
\text { hipocrómica } \\
\text { Sin anemia }\end{array}$ & $\begin{array}{l}10 \% \\
90 \%\end{array}$ & $\begin{array}{l}\text { Con aumento } \\
\text { Normal }\end{array}$ & $\begin{array}{l}62 \% \\
12 \% \\
26 \%\end{array}$ & $\begin{array}{l}\text { Relacionados } \\
\text { No relacionados } \\
\text { Asintomático }\end{array}$ \\
\hline 1 & $\begin{array}{l}\text { Heterocigoto } \\
\qquad \mathrm{A}-\mathrm{C}\end{array}$ & $100 \%$ & $\begin{array}{l}\text { Hemoglobi- } \\
\text { nopatía C }\end{array}$ & $100 \%$ & Sin anemia & $100 \%$ & Normal & $100 \%$ & Relacionados \\
\hline 3 & $\begin{array}{l}\text { Heterocigoto } \\
\text { S-C }\end{array}$ & $100 \%$ & $\begin{array}{l}\text { Hemoglobi- } \\
\text { nopatía C }\end{array}$ & $\begin{array}{l}66,67 \% \\
33,33 \%\end{array}$ & $\begin{array}{l}\text { Microcítica } \\
\text { hipocrómica } \\
\text { Sin anemia }\end{array}$ & $100 \%$ & Normal & $\begin{array}{l}66,67 \% \\
33,33 \%\end{array}$ & $\begin{array}{l}\text { Relacionados } \\
\text { Asintomático }\end{array}$ \\
\hline 7 & $\begin{array}{l}\text { Heterocigoto } \\
\text { S-B talasemia }\end{array}$ & $\begin{array}{l}71,43 \% \\
14,28 \% \\
14,28 \%\end{array}$ & $\begin{array}{l}\text { con Hemoglo- } \\
\text { binopatía S } \\
\text { Otras anemias } \\
\text { Diagnóstico } \\
\text { no relacionado }\end{array}$ & $\begin{array}{l}28,57 \% \\
14,29 \% \\
57,14 \%\end{array}$ & $\begin{array}{l}\text { Microcítica } \\
\text { hipocrómica } \\
\text { Microcítica } \\
\text { normocrómica } \\
\text { Sin anemia }\end{array}$ & $\begin{array}{l}42,86 \% \\
57,14 \%\end{array}$ & $\begin{array}{l}\text { con aumento } \\
\text { normal }\end{array}$ & $\begin{array}{l}71,43 \% \\
28,57 \%\end{array}$ & $\begin{array}{l}\text { Relacionados } \\
\text { Asintomático }\end{array}$ \\
\hline 18 & $\begin{array}{l}\text { Rasgo } \\
\text { falciforme } \\
\text { (Hb A-S) }\end{array}$ & $\begin{array}{l}77,78 \% \\
5,56 \% \\
16,67 \%\end{array}$ & $\begin{array}{l}\text { Hemoglobi- } \\
\text { nopatía S. } \\
\text { Otras anemias } \\
\text { Diagnóstico } \\
\text { no relacionado }\end{array}$ & $\begin{array}{l}5,56 \% \\
11,11 \% \\
83,33 \%\end{array}$ & $\begin{array}{l}\text { Microcítica } \\
\text { hipocrómica } \\
\text { Normocítica } \\
\text { normocrómica } \\
\text { Sin anemia }\end{array}$ & $\begin{array}{l}11,11 \% \\
88,89 \%\end{array}$ & $\begin{array}{l}\text { con aumento } \\
\text { normal }\end{array}$ & $\begin{array}{l}83,33 \% \\
5,56 \% \\
11,11 \%\end{array}$ & $\begin{array}{l}\text { Relacionados } \\
\text { No relacionados } \\
\text { Asintomático }\end{array}$ \\
\hline
\end{tabular}

\section{Discusión}

La hemoglobina humana puede presentar alteraciones conocidas como hemoglobinopatías, ocasionadas por trastornos hereditarios autosómicos. Estas variantes de la hemoglobina pueden ser de dos tipos: cualitativas o estructurales y cuantitativas, y estas son cada vez más frecuentes en la población en consecuencia del aumento de las corrientes migratorias de los seres humanos.

La presencia global de variantes de hemoglobina en Colombia fue de $38,83 \%$, siendo la talasemia la hemoglobinopatía cuantitativa más frecuente. En cuanto a las variantes cualitativas, el rasgo falciforme lidera la lista tal como se observó en investigaciones previas ${ }^{2,4}$. El alto porcentaje de variantes de hemoglobinas en este estudio puede explicarse debido a que se incluyeron tanto hemoglobinopatías cualitativas como cuantitativas, mientras que en la mayoría de estudios reportados se hace principalmente la detección de variantes de hemoglobina cualitativas. Por otra parte, el trabajo realizado en Colombia por Pereira y Sáenz, en $1996^{16}$, reportó un porcentaje similar $(45,26 \%)$, en el que además de detectar hemoglobinas estructurales o cualitativas también se detectaron talasemias compuestas. Adicionalmente, las 1.407 muestras del estudio no fueron escogidas al azar en población sana, por el contrario, todas provenían de individuos con sospecha de hemoglobinopatías.

La región colombiana con mayor número de casos remitidos con alteraciones de la hemoglobina fue la región Andina, seguida por la Amazonia y la Orinoquía, lo cual es consistente con los reportes de la Organización Internacional para las Migraciones (OIM) en los que el mayor porcentaje de asentamientos de inmigrantes en Colombia se ubica en esta región, pudiendo alterar la homogeneidad de la población ${ }^{17}$.

Aunque se define como inmigrante al movimiento de personas no nacionales que ingresa a 
un país con el objetivo de establecerse allí, es importante destacar también que el conflicto armado del país promueve forzosamente desplazamientos a las principales ciudades colombianas ${ }^{18,19}$.

La población con menores casos de hemoglobinopatías fue la región afro-colombiana que se ubica al norte de la costa pacífica y la isla de San Andrés, lo cual se puede explicar por el bajo volumen de muestras procesadas provenientes de dicha población.

Las especialidades que más remitieron para evaluación de hemoglobinas fueron hematooncología y medicina interna, lo que sugiere el buen manejo y adecuado algoritmo de diagnóstico que se le da a pacientes con sospecha de hemoglobinopatías en dichas disciplinas. El cuadro clínico inicial (generalmente signo o síntoma) es muy importante, dado que forma parte de estos mismos criterios clínicos. Sin embargo, como el servicio de hematooncología representa una supra-especialidad, generalmente, los pacientes son derivados de la consulta de atención primaria. Se incluyeron síntomas y signos, así como paraclínicos iníciales como datos orientadores para la generación de la evaluación al estudio de la hemoglobinopatía e inducir un diagnóstico presuntivo eficaz.

Dentro de las técnicas disponibles para su detección, se encuentran la EC y la electroforesis en gel de agarosa. Los resultados de su comparación y correlación fueron considerados adecuados en tres de los cuatro tipos de hemoglobinas analizadas (A, F, S), mientras que para A2 se obtuvo baja correlación. Este hecho se puede explicar por la capacidad que tiene la EC de detectar variantes adicionales de hemoglobina ${ }^{20}$. La concordancia por coeficiente de Pearson fue considerada buena, mientras que por índice Kappa la correlación fue superior. Esta variación puede estar dada por la naturaleza de los análisis, mientras que el coeficiente de Pearson evalúa variable por variable, confrontando el valor porcentual de cada tipo de hemoglobina, el índice Kappa calcula generalmente concordancia para variables dicotómicas.

En cuanto a comparación técnica, cuando se empleó EC en el caso particular de la baja correlación de $\mathrm{Hb}$ A2 y aumento de los valores de la misma, se evidenció un resultado similar al obtenido por Keren y cols ${ }^{21}$, compararon EC y HPLC para el diagnóstico de hemoglobinopatías, obteniendo ligero aumento de $\mathrm{Hb} \mathrm{A} 2$ por el primer método.

La variación de hemoglobina A2 en electrofo- resis en geles de agarosa se ha descrito en estudios como el de Clarke y Higgins, donde comparan EC y HPLC y concluyen que este tipo de electroforesis carece de exactitud y precisión al detectar hemoglobina $\mathrm{A} 2^{22,23}$.

La EC ofrece ventajas operativas frente a la electroforesis en gel de agarosa: mínima cantidad de muestra, realización de diluciones precisas, no requiere lavado de eritrocitos, rapidez y automatización. La electroforesis en gel de agarosa detecta las variantes de hemoglobina en dos etapas: $\mathrm{pH}$ ácido y $\mathrm{pH}$ alcalino. Para la elección de la técnica debe tenerse en cuenta el tipo de laboratorio, el número de muestras a procesar y la oportunidad requerida en entrega de resultados.

\section{Conclusiones}

La frecuencia de hemoglobinas variantes en las poblaciones fue alta, lo cual constituye un problema de salud pública, por lo que se sugiere implementar planes y programas que enfaticen el manejo de esta problemática. En Colombia hay regiones de alta densidad poblacional de afro colombianos y constante migración, lo cual plantea la necesidad de establecer estrategias para la identificación temprana de los portadores y enfermos con propósitos de prevención.

La variante de hemoglobina más frecuente fue $\mathrm{Hb}$ A/Aumento de A2 y la hemoglobinopatía estructural fue el rasgo falciforme. Finalmente, la EC y la electroforesis en agarosa fueron consideradas técnicas con buena correlación y concordancia para la detección de hemoglobinas, con la posibilidad de diferenciar mayor cantidad de variantes en la electroforesis capilar.

Agradecimientos: Al Dr. Jorge Salinas MD, hemato-oncológo y al Dr. Sebastián Giraldo MD, internista, por su colaboración en la clasificación de las variantes clínicas y de las hemoglobinopatías para el desarrollo de este trabajo.

\section{Referencias}

1. Villarreal ME, Bermúdez A. Tamizaje para hemoglobinopatías en la Isla de San Andrés. En: Colombia Informe Quincenal-Epidemiológico Nacional ISSN: 0122-9907 en: Instituto Nacional de Salud 2001. fasc. 15 p. 244-6. 
2. Cuellar-Ambrosi F, Mondragón MC, Figueroa M, Prehu C, Galacteros F, Ruiz-Linares A. Sicklecellanaemia and Beta-globin gene clusterhaplotypes in Colombia. Hemoglobin 2000; 24: 221-5.

3. Bernal M, Collazos A, Bonilla RD, Tascón EP. Determination of the prevalence of hemoglobin S, C, D, and G in neonates from Buenaventura. Colomb Med 2010; 41: 141-7.

4. Alvear C, Barboza M, Alayón AN, Viola M, Araque LM. Pilot study of hemoglobinopathies in newborns of the Rafael Calvo maternity clinic of Cartagena, Colombia. Colomb Med 2012; (43): 197-8.

5. Silva JR, Malambo D, Silva DF, Fals E, Fals O, Rey J. Tamizaje de hemoglobinopatías en una muestra de la población infantil de Cartagena. Pediatría 1998; 33 (2): 86-9.

6. Satizábal JM. Prevalencia de Hemoglobinopatías en el casco urbano de la ciudad de Buenaventura, Memorias Reunión Anual TSH Neonatal, Instituto Nacional De Salud 2010; 16-8.

7. Rosero MJ, Bermúdez AJ. Análisis de hemoglobinopatías en regiones afrocolombianas usando muestras de sangre seca de cordón umbilical. Acta Med Colomb 2012; (37): 118-24.

8. Bernal M. del P, Giraldo A, Bermúdez AJ, Moreno E. Estudio de la frecuencia de hemoglobinopatías en las islas de San Andrés y Providencia. Biomédica 1995; 15: 5-9.

9. Restrepo F, Loaiza N, Arrubla M, Cossio S, Ordoñez J. Estudio de la prevalencia de anemia falciforme y otras hemoglobinopatías en población de recién nacidos del Área Metropolitana de Medellín [En línea]. Memorias taller anual TSH Neonatal; 2010, Nov 16-18, 237-9 [consultado el 25 de noviembre de 2014]. Disponible en: http://www.ins.gov.co/?idcategoria=82033\#

10. Freifelder D. Técnicas de bioquímica y biología molecular. Barcelona. Ed. Reverté 2003; 237-54.

11. Osatinsky R. ¿Qué es la electroforesis capilar? Bioquímica y Patología Clínica 2007; 71 (2): 60-6.

12. Ji-Eun K, Bo-Ram K, Kwang-Sook W, Jeong-Man K, Joo-In P, Jin-Yeong H. Comparison of Capillary Electrophoresis with Cellulose Acetate Electrophoresis for the Screening of Hemoglobinopathies. Korean J Lab Med 2011; 31 (4): 238-43.
13. Barrera P. Desarrollan técnica para diagnosticar "mala" sangre. Universidad Nacional de Colombia. UN periódico. 2009 Dic 12; Sección Ciencia \& Tecnología. Impreso $\mathrm{N}^{\circ} 129$.

14. Tefferi A. Anemia in adults: a contemporary approach to diagnosis. Mayo Clin Proc 2003; 1274-80.

15. Paredes M, Galindo A, Bernal M, Ávila S, Andrade D, Vergara C. Analysis of the CODIS autosomal STR loci in four main Colombian regions. Forensic Sci Int 2003; 137 (1): 67-73.

16. Pereira FD, Sáenz I. Hemoglobinopatías en niños. Colombia Med 1996; (27): 147-8.

17. Ramírez C, Zuluaga M, Perilla C. Perfil migratorio de Colombia [En línea]. [consultado el 25 de noviembre de 2014]. Disponible en: Organización Internacional para las migraciones: http://www.retornovoluntariolatam. org/sites/default/files/documentos/perfil_migratorio_colombia_2012_oim.pdf

18. International Organization for Migration (IOM). términos clave de migración Route des Morillons [En línea]. Suiza [consultado el 25 de septiembre de 2014]. Disponible en: http://www.iom.int/cms/es/sites/iom/ home/about-migration/key-migration-terms-1.ht$\mathrm{ml}$ Inmigraci $\% \mathrm{C} 3 \% \mathrm{~B} 3 \mathrm{n}$

19. Correa A, Palacio J, Sandro Jiménez S, Díaz MR. Desplazamiento interno forzado-Restablecimiento urbano e identidad social. $1^{\text {a }}$ ed. Colombia. 2009; 5-25.

20. Instituto de Referencia Andino. Novedades en el diagnóstico electroforético de las hemoglobinopatías. Boletín informativo 2011; 5 (2).

21. Keren DF, Hedstrom D, Gulbranson R, Ou C-N, Bak R. Comparison of Sebia Capillarys capillary electrophoresis with the Primus high-pressure liquid chromatography in the evaluation of hemoglobinopathies. Am J Clin Pathol 2008; 130: 824-31.

22. Clarke G, Higgins TN. Laboratory investigation of hemoglobinopathies and thalassemia: review and update. Clin Chem 2000; 46: 1284-90.

23. Winichagoon P, Svasti S, Munkongdee T, Chaiya W, Boonmongkol P, Chantrakul N, et al. Rapid diagnosis of thalassemias and other hemoglobinopathies by capillary electrophoresis system. Translational Research 2008; 152 (4): 180-3. 